\title{
Intermediate spaces, Gaussian probabilities and exponential tightness
}

\section{Paolo Baldi ${ }^{1}$}

Received: 6 May 2020 / Accepted: 19 March 2021 / Published online: 8 April 2021

(c) The Author(s) 2021

\begin{abstract}
We prove the existence of an intermediate Banach space between the space where the Gaussian measure lives and its RKHS, thus extending what happens with Wiener measure, where the intermediate space can be chosen as a space of Hölder paths. From this result, it is very simple to deduce a result of exponential tightness for Gaussian probabilities.
\end{abstract}

Keywords Gaussian probabilities · Exponential tightness · Interpolation theory

Mathematics Subject Classification $60 \mathrm{~F} 10 \cdot 60 \mathrm{~B} 12$

\section{Introduction}

Let $E=\mathscr{C}_{0}\left([0, T], \mathbb{R}^{m}\right)$ be the space of continuous $\mathbb{R}^{m}$-valued paths starting at 0 and endowed with the sup norm and let $\mu$ be the Wiener measure on it. It is well known that the Reproducing Kernel Hilbert Space (RKHS) of this Gaussian probability is the space $\mathscr{H}=H_{0}^{1}([0, T])$ of the paths $\gamma$ vanishing at 0 , that are absolutely continuous and have a square integrable derivative.

Let us denote by $\mathscr{C}_{\alpha}^{0} \subset E$ the space of the paths that are $\alpha$-Hölder continuous and whose modulus of continuity

$$
\omega(\delta)=\sup _{\substack{0 \leq s<t \leq T \\|t-s| \leq \delta}}|\gamma(t)-\gamma(s)|
$$

is such that

$$
\lim _{\delta \rightarrow 0+} \frac{\omega(\delta)}{\delta^{\alpha}}=0
$$

This space (whose elements are sometimes called the "small" $\alpha$-Hölder continuous functions) is separable and it is also well known (thanks to Kolmogorov's continuity theorem)

Paolo Baldi

baldi@mat.uniroma2.it

1 Dipartimento di Matematica, Università di Roma “Tor Vergata”, Rome, Italy 
that, for $0<\alpha<\frac{1}{2}, \mu\left(\mathscr{C}_{\alpha}^{0}\right)=1$ (whereas $\mu(\mathscr{H})=0$ ). It is also well known that, still for $0<\alpha<\frac{1}{2}$,

$$
E \hookleftarrow \mathscr{C}_{\alpha}^{0} \hookleftarrow \mathscr{H}
$$

the embeddings being compact.

We are concerned with the question whether the existence of such an "intermediate" space is a general fact, i.e. true for every centered Gaussian probability on a separable Banach space.

More precisely we prove the following result.

Theorem 1.1 Let $E$ be a separable Banach space, $\mu$ a centered Gaussian probability on $E$ and $\mathscr{H}$ the corresponding RKHS. Then there exists a Banach space $\widetilde{E}$, separable and such that

(a) $\mu(\widetilde{E})=1$ and

(b) the embeddings

$$
E \hookleftarrow \widetilde{E} \hookleftarrow \mathscr{H}
$$

are compact.

We shall even prove that there are infinitely many such spaces. We shall call "intermediate space" any separable Banach space satisfying (a) and (b) of Theorem 1.1.

The proof of Theorem 1.1 is the object of Sect. 3. Of course Theorem 1.1 is obvious if $E$ is finite dimensional, as then we can choose $E=\widetilde{E}=\mathscr{H}$. Therefore in the sequel we implicitly assume that $E$ is infinite dimensional.

In the proof we shall also assume that $E=\operatorname{supp}(\mu)$. Otherwise just consider $\operatorname{supp}(\mu)$ instead of $E$.

This investigation was motivated by an application to the Large Deviations of the sequence of probabilities $\left(\mu_{\varepsilon}\right)_{\varepsilon}, \mu_{\varepsilon}$ being the image of $\mu$ through the map $x \mapsto \varepsilon x$. The property of exponential tightness is a key step in the proof of these estimates. One remarks that its proof in the case of Wiener measure is particularly simple and is based, besides Fernique's theorem, on the existence of the spaces of Hölder continuous functions, which are intermediate spaces. Thanks to Theorem 1.1 the same, simple proof of exponential tightness for the Wiener measure works for a general Gaussian probability on a separable Banach space as developed in Sect. 2.

Section 4 is devoted to comments and complements.

The proof of Theorem 1.1 is largely inspired to the arguments of the fundamental papers of L.Gross [7, 8].

\section{Motivation: exponential tightness of Gaussian measures}

Let $\mu$ be a centered Gaussian probability on the separable Banach space $E$ and let $\mu_{\varepsilon}$ be its image through the map $x \mapsto \varepsilon x$ as above.

The Large Deviations properties of the family $\mu_{\varepsilon}$ as $\varepsilon \rightarrow 0$ are well understood since a long time (see [4] $\$ 5$ e.g.). One of the main steps in this investigation is to prove that the 
family $\left(\mu_{\varepsilon}\right)_{\varepsilon}$ is exponentially tight at speed $\varepsilon \mapsto \varepsilon^{2}$, i.e. that for every $R>0$ there exists a compact set $K_{R} \subset E$ such that

$$
\limsup _{\varepsilon \rightarrow 0} \varepsilon^{2} \log \mu_{\varepsilon}\left(K_{R}^{c}\right) \leq-R .
$$

This fact follows immediately from Theorem 1.1: let us denote \|\|$_{i}$ the norm of the intermediate space $\widetilde{E}$, as \|\|$_{i}$ is $\mu$-a.s. finite (this is a) of Theorem 1.1), by Fernique's theorem ( [5, 6]), for some $\rho>0$, we have

$$
\int_{E} \mathrm{e}^{\rho\|x\|_{i}^{2}} \mathrm{~d} \mu(x):=C_{\rho}<+\infty .
$$

Let $K_{R}$ denote the ball of radius $\sqrt{R / \rho}$ of $\widetilde{E}$, which is compact in $E$. From

$$
C_{\rho} \geq \int_{K_{R}^{c} / \varepsilon} \mathrm{e}^{\rho\|x\|_{i}^{2}} \mathrm{~d} \mu(x) \geq \mu\left(\frac{1}{\varepsilon} K_{R}^{c}\right) \mathrm{e}^{R / \varepsilon^{2}}
$$

we deduce

$$
\limsup _{\varepsilon \rightarrow 0} \varepsilon^{2} \log \mu_{\varepsilon}\left(K_{R}^{c}\right)=\limsup _{\varepsilon \rightarrow 0} \varepsilon^{2} \log \mu\left(\frac{1}{\varepsilon} K_{R}^{c}\right) \leq-R
$$

i.e. (2.1).

\section{Proof of the main result}

From now on $\mu$ will denote a Gaussian probability on the infinite dimensional separable Banach space $E$ as in the introduction, \|\| being the norm of the Banach space $E$.

If we denote by $E^{\prime}$ the dual of $E$, then to every continuous functional $\xi \in E^{\prime}$ we can associate the r.v. $(E, \mu) \rightarrow \mathbb{R}$ defined as $x \mapsto\langle\xi, x\rangle$. Let $E_{\mu}^{\prime}$ be the completion of $E^{\prime}$ in $L^{2}(\mu)$. This is a separable Hilbert space which is also a Gaussian space.

For every $g \in E_{\mu}^{\prime}$ the vectors

$$
h=\int_{E} x g(x) \mathrm{d} \mu(x)
$$

form a vector space $\mathscr{H} \subset E$ which, endowed with the scalar product

$$
\left\langle h_{1}, h_{2}\right\rangle_{\mathscr{C}}=\int_{E} g_{1}(x) g_{2}(x) \mathrm{d} \mu(x)
$$

for

$$
h_{1}=\int_{E} x g_{1}(x) \mathrm{d} \mu(x), \quad h_{2}=\int_{E} x g_{2}(x) \mathrm{d} \mu(x),
$$

is an Hilbert space $\mathscr{H}$ isometric to $E_{\mu}^{\prime}$. Remark that (3.2) can also be written $h=\mathrm{E}[X g(X)]$, $X$ denoting an $E$-valued r.v. having law equal to $\mu$. $\mathscr{H}$ is called the Reproducing Kernel Hilbert Space (RKHS) of $\mu$.

For more details on the structure of Gaussian probabilities see [9] or the very nice and very short presentation in $\$ 2$ of [3]. 
Proof of Theorem 1.1 Recall that we assume $E$ to be infinite dimensional.

(a) First step: construction of $\widetilde{E}$.

Let $(\Omega, \mathscr{F}, \mathrm{P})$ be a probability space and $X: \Omega \rightarrow E$ a Gaussian r.v. having distribution $\mu$. Let $\left(g_{n}\right)_{n}$ be an orthonormal system of the Hilbert space $E_{\mu}^{\prime}$. This forms also a sequence of independent $N(0,1)$-distributed r.v.'s. Let $e_{n}=\mathrm{E}\left[X g_{n}(X)\right] . e_{n} \in E$ and $\left(e_{n}\right)_{n}$ is an orthonormal system of the RKHS $\mathscr{H}$.

Then it is well known (see Proposition 3.6 p. 64 in [9]) that the sequence

$$
X_{n}=\sum_{j=1}^{n} g_{j} e_{j}
$$

is a square integrable $E$-valued martingale converging a.s. and in $L^{2}$ to $X$. Hence

$$
\lim _{n \rightarrow \infty} \mathrm{E}\left(\left\|\sum_{j=n}^{\infty} g_{j} e_{j}\right\|^{2}\right)=0 \text {. }
$$

Let $\alpha>0$ be fixed and let $\left(n_{k}\right)_{k}$ be an increasing sequence of integers such that $n_{0}=0$ and

$$
\mathrm{E}\left(\left\|\sum_{j=n_{k}+1}^{\infty} g_{j} e_{j}\right\|^{2}\right) \leq 2^{-k(3+2 \alpha)} .
$$

Let $\mathscr{H}_{k}=\operatorname{span}\left(e_{n_{k}+1}, \ldots, e_{n_{k+1}}\right)$ and let $Q_{k}$ be the projector $\mathscr{H} \rightarrow \mathscr{H}_{k}$. Let, for the vector $x=\sum_{n=1}^{\infty} \alpha_{n} e_{n} \in \mathscr{H}$,

$$
\|x\|_{i}:=\sum_{k=0}^{\infty} 2^{k \alpha}\left\|\sum_{j=n_{k}+1}^{n_{k+1}} \alpha_{j} e_{j}\right\|=\sum_{k=1}^{\infty} 2^{k \alpha}\left\|Q_{k}(x)\right\| .
$$

\|\|$_{i}$ is a norm on $\mathscr{H}$. Actually Lemma 3.2 states that $\|x\|_{i}<+\infty$ for every $x \in \mathscr{H}$ while subadditivity and positive homogeneity are immediate. Let

$$
W_{k}:=\sum_{j=n_{k}+1}^{n_{k+1}} g_{j} e_{j}
$$

The $E$-valued r.v.'s $W_{k}$ are Gaussian and independent. Remark that, with our choice of the numbers $n_{k}$, thanks to the Markov inequality we have

$$
\mathrm{P}\left(2^{k \alpha}\left\|W_{k}\right\| \geq 2^{-k}\right) \leq 2^{2 k(1+\alpha)} \mathrm{E}\left(\left\|W_{k}\right\|^{2}\right) \leq 2^{2 k(1+\alpha)} \mathrm{E}\left(\left\|\sum_{j=n_{k}+1}^{\infty} g_{j} e_{j}\right\|^{2}\right) \leq 2^{-k} .
$$

We can now define $\widetilde{E}=$ the completion of $\mathscr{H}$ with respect to the norm \|\|$_{i}$. Remark that, as for $x \in \mathscr{H}$

$$
\|x\|_{i}=\sum_{k=1}^{\infty} 2^{k \alpha}\left\|Q_{k}(x)\right\| \geq \sum_{k=1}^{\infty}\left\|Q_{k}(x)\right\| \geq\left\|\sum_{k=1}^{\infty} Q_{k}(x)\right\|=\|x\|,
$$

we have $\widetilde{E} \subset E$. It is also obvious that $\widetilde{E}$ is dense in $E$, as it contains $\mathscr{H}$ which is itself dense in $E$. (b) Second step: $\mu(\widetilde{E})=1$. Let 


$$
Y_{k}=X_{n_{k}}=\sum_{j=1}^{n_{k}} g_{j} e_{j}
$$

and let us prove that $\left(Y_{k}\right)_{k}$, as a sequence of $\widetilde{E}$-valued r.v.'s, converges in probability. We proceed quite similarly as in the proof of the subsequent Lemma 3.2. Let $\varepsilon>0$. If $p_{0}$ is such that $2^{-p_{0}}<\frac{\varepsilon}{2}$, then for $p_{0} \leq \ell<r$,

$$
\begin{aligned}
& \mathrm{P}\left(\left\|Y_{r}-Y_{\ell}\right\|_{i}>\varepsilon\right) \leq \mathrm{P}\left(\sum_{k=\ell+1}^{r} 2^{\alpha k}\left\|W_{k}\right\|>\sum_{k=\ell+1}^{r} 2^{-k}\right) \\
& \leq \sum_{k=\ell+1}^{r} \mathrm{P}\left(2^{\alpha k}\left\|W_{k}\right\|>2^{-k}\right) \leq \sum_{k=p_{0}+1}^{\infty} 2^{-k} \leq 2 \cdot 2^{-p_{0}}=\varepsilon .
\end{aligned}
$$

Therefore $\left(Y_{k}\right)_{k}$ is a Cauchy sequence in probability in $\widetilde{E}$, hence it converges, in probability, to some $E$-valued r.v. $\widetilde{X}$. As $\widetilde{E} \subset E$ and its topology is stronger, $\left(Y_{k}\right)_{k}$ also converges in probability in $E$. But we know already that $\left(Y_{k}\right)_{k}$ in $E$ converges to a r.v. $X$ having law $\mu$. Hence $\widetilde{X}=X$ a.s. and $\mu(\widetilde{E})=\mathrm{P}(X \in \widetilde{E})=\mathrm{P}(\widetilde{X} \in \widetilde{E})=1$.

(c) Step three: the embedding $E \hookleftarrow \widetilde{E}$ is compact.

Let $\left(x_{p}\right)_{p}$ be a bounded sequence in $\widetilde{E}$ and $\left(z_{p}\right)_{p_{\widetilde{E}}} \subset \mathscr{H}$ another sequence such that $\left\|x_{p}-z_{p}\right\|_{i}<2^{-p}$, which is possible as $\mathscr{H}$ is dense in $\widetilde{E}$. Let $M$ be such that $\left\|z_{p}\right\|_{i} \leq M$ for every $p$. As the projectors $Q_{k}$ are finite dimensional, for every $k$ there exists a subsequence $\left(p_{r}^{(k)}\right)_{r}$ such that $\left\|Q_{k} z_{p_{r}^{(k)}}-y^{(k)}\right\|_{i} \rightarrow 0$ for some vector $y^{(k)} \in \mathscr{H}_{k}$, i.e. of the form

$$
y^{(k)}=\sum_{m=n_{k}+1}^{n_{k+1}} \alpha_{m} e_{m} .
$$

By the diagonal argument, there exists a subsequence $\left(p_{r}^{\prime}\right)_{r}$ such that $\left\|Q_{k} z_{p_{r}^{\prime}}-y^{(k)}\right\|_{i} \rightarrow 0$ as $r \rightarrow \infty$ for every $k$. Let now $\varepsilon>0$ be fixed. We have, for every positive integer $k_{0}$,

$$
\begin{aligned}
\left\|z_{p_{r}^{\prime}}-z_{p_{\ell}^{\prime}}\right\| & =\left\|\sum_{k=1}^{\infty} Q_{k}\left(z_{p_{r}^{\prime}}-z_{p_{\ell}^{\prime}}\right)\right\| \\
& \leq\left\|\sum_{k=1}^{k_{0}} Q_{k}\left(z_{p_{r}^{\prime}}-z_{p_{\ell}^{\prime}}\right)\right\|+\left\|\sum_{k=k_{0}+1}^{\infty} Q_{k}\left(z_{p_{r}^{\prime}}-z_{p_{\ell}^{\prime}}\right)\right\| .
\end{aligned}
$$

We first choose $k_{0}$ so that $M 2^{-\alpha k_{0}}<\frac{\varepsilon}{3}$, so that

$$
\begin{aligned}
\sum_{k=k_{0}+1}^{\infty}\left\|Q_{k}\left(z_{p_{r}^{\prime}}-z_{p_{e}^{\prime}}\right)\right\| & \leq 2^{-\alpha k_{0}} \sum_{k=k_{0}+1}^{\infty} 2^{\alpha k}\left\|Q_{k}\left(z_{p_{r}^{\prime}}-z_{p_{e}^{\prime}}\right)\right\| \\
& \leq 2^{-\alpha k_{0}}\left\|z_{p_{r}^{\prime}}-z_{p_{e}^{\prime}}\right\|_{i} \\
& \leq 2^{-\alpha k_{0}} \cdot 2 M \leq \frac{2}{3} \varepsilon
\end{aligned}
$$

and then $p_{0}$ so that, for $r, \ell \geq p_{0}$

$$
\left\|\sum_{k=1}^{k_{0}} Q_{k}\left(z_{p_{r}^{\prime}}-z_{p_{\ell}^{\prime}}\right)\right\| \leq \frac{\varepsilon}{3} .
$$


Therefore $\left(z_{p_{r}^{\prime}}\right)_{r}$ is a Cauchy sequence in $E$, hence also $\left(x_{p_{r}^{\prime}}\right)_{r}$ which proves the compactness of the embedding $\widetilde{E} \hookrightarrow E$.

d) Last step $\widetilde{E} \hookleftarrow \mathscr{H}$ is compact. This is immediate as, $\widetilde{E}$ being dense in $E$, $\mathscr{H}$ is also the RKHS of the Gaussian probability $\mu$ on $\widetilde{E}$. Such an embedding is always compact.

Lemma 3.1 Let $\mathscr{K}$ be a finite dimensional Hilbert space and $\mathscr{F} \subset \mathscr{K}$ a subspace and let us denote by $v$ and $v^{\prime}$ the standard $N(0, I)$ distributions on $\mathscr{K}$ and $\mathscr{F}$ respectively. Let $B \subset \mathscr{K}$ be a convex, centrally symmetric, convex set. Then

$$
\nu(B) \leq v^{\prime}(\mathscr{F} \cap B) .
$$

For the proof of Lemma 3.1 the reader is directed to [7] as this is a weaker version of Lemma 4.1 there.

Lemma $3.2\|x\|_{i}<+\infty$ for every $x \in \mathscr{H}$.

Proof Let us first prove that the sequence of real r.v.'s

$$
Z_{n}=\sum_{k=1}^{n} 2^{k \alpha}\left\|W_{k}\right\|
$$

converges in probability. Let $\varepsilon>0$ and $p_{0}$ such that $2^{-p_{0}}<\frac{\varepsilon}{2}$. Remark that this implies $\varepsilon>\sum_{k=p_{0}+1}^{\infty} 2^{-k}$. For $p_{0} \leq n<m$, we have thanks to (3.7)

$$
\begin{aligned}
\mathrm{P}\left(\left|Z_{n}-Z_{m}\right|>\varepsilon\right) & =\mathrm{P}\left(\sum_{k=n+1}^{m} 2^{k \alpha}\left\|W_{k}\right\|>\varepsilon\right) \leq \mathrm{P}\left(\sum_{k=n+1}^{m} 2^{k \alpha}\left\|W_{k}\right\|>\sum_{k=n+1}^{m} 2^{-k}\right) \\
& \leq \sum_{k=n+1}^{m} \mathrm{P}\left(2^{k \alpha}\left\|W_{k}\right\|>2^{-k}\right) \leq \sum_{k=n+1}^{m} 2^{-k} \leq \sum_{k=p_{0}+1}^{\infty} 2^{-k}=2 \cdot 2^{-p_{0}}<\varepsilon .
\end{aligned}
$$

Hence $\left(Z_{n}\right)_{n}$ is a Cauchy sequence in probability and converges in probability to some real r.v. $Z$.

Let us prove that, for every $\varepsilon>0$, we have $\mathrm{P}(Z<\varepsilon)>0$. Let

$$
Z_{N}=\sum_{k=1}^{N} 2^{k \alpha}\left\|W_{k}\right\|, \quad Z-Z_{N}=\sum_{k=N+1}^{\infty} 2^{k \alpha}\left\|W_{k}\right\| .
$$

We have $\mathrm{P}\left(Z_{N}<\frac{\varepsilon}{2}\right)>0$, as $Z_{N}$ depends only on the modulus of finitely many r.v.'s $W_{k}$ each of them being Gaussian and with values in a finite dimensional vector space. Moreover let $N$ be large enough so that $\mathrm{P}\left(Z-Z_{N}<\frac{\varepsilon}{2}\right)>0$. As the r.v.'s $Z-Z_{N}$ and $Z_{N}$ are independent (they depend on different $W_{k}$ 's)

$$
\mathrm{P}(Z<\varepsilon) \geq \mathrm{P}\left(Z-Z_{N}<\frac{\varepsilon}{2}, Z_{N}<\frac{\varepsilon}{2}\right)=\mathrm{P}\left(Z-Z_{N}<\frac{\varepsilon}{2}\right) \mathrm{P}\left(Z_{N}<\frac{\varepsilon}{2}\right)>0 .
$$

Finally, let us assume that there exists $x=\sum_{i=1}^{\infty} \alpha_{j} e_{j} \in \mathscr{H}$ such that $\|x\|_{i}=+\infty$ and let us prove that this is absurd. Of course we can assume $\|x\|_{\mathscr{H}}=1$. Let us consider the seminorms on $\mathscr{H}$ 


$$
\|z\|_{k}=\sum_{j=1}^{k} 2^{j \alpha}\left\|Q_{j}(z)\right\|
$$

so that $\lim _{k \rightarrow \infty}\|z\|_{k}=\|z\|_{i}$. Let $\mathscr{K}_{k}=\operatorname{span}\left(e_{1}, \ldots, e_{n_{k}}\right)$ so that the r.v. $X_{n_{k}}$ of (3.3) takes values in $\mathscr{K}_{k}$. Let $x^{(k)}=\sum_{i=1}^{n_{k}} \alpha_{j} e_{j}$ be the projection of $x$ on $\mathscr{K}_{k}$. Remark that $\|x\|_{k}=\left\|x^{(k)}\right\|_{k}$. We apply Lemma 3.1 considering the convex set

$$
B=\left\{z \in \mathscr{K}_{k},\|z\|_{k} \leq a\right\}
$$

and $\mathscr{F}=\operatorname{span}\left(x^{(k)}\right)$. Let $\xi_{k}=\sqrt{\alpha_{1}^{2}+\cdots+\alpha_{n_{k}}^{2}}$, so that the vector $\frac{x^{(k)}}{\xi_{k}}$ has modulus 1 in $\mathscr{H}$ and the r.v.

$$
g:=\frac{1}{\xi_{k}} \sum_{j=1}^{n_{k}} \alpha_{j} g_{j}
$$

is $N(0,1)$-distributed. Hence the r.v.

$$
\frac{1}{\xi_{k}} \sum_{j=1}^{n_{k}} \alpha_{j} g_{j} \cdot \frac{x^{(k)}}{\xi_{k}}
$$

is $N(0,1)$-distributed and $\mathscr{F}$-valued.

Let $a$ be a continuity point of the partition function of $Z$. Thanks to Lemma 3.1, as $\xi_{k} \rightarrow\|x\|_{\mathscr{H}}=1$ and we assume $\|x\|_{i}=+\infty$, we have

$$
\begin{aligned}
\mathrm{P}(Z \leq a) & =\lim _{k \rightarrow \infty} \mathrm{P}\left(\left\|\sum_{j=1}^{n_{k}} g_{j} e_{j}\right\|_{i} \leq a\right) \leq \lim _{k \rightarrow \infty} \mathrm{P}\left(\left|\frac{1}{\xi_{k}} \sum_{j=1}^{n_{k}} \alpha_{j} g_{j}\right| \frac{\|x\|_{k}}{\xi_{k}} \leq a\right) \\
& =\lim _{k \rightarrow \infty} \mathrm{P}\left(|g| \leq \frac{a \xi_{k}}{\|x\|_{k}}\right)=0
\end{aligned}
$$

which is in contradiction with (3.9) and completes the proof of Lemma 3.2.

\section{Remarks and complements}

Remark 4.1 In fact we have proved the existence on infinitely many intermediate spaces $\widetilde{E}$ between $E$ and $\mathscr{H}$ (recall that we assume that $E$ is infinite dimensional). Actually the argument above can be repeated in order to construct a subsequent intermediate space $\widetilde{E}_{1}$ between $\mathscr{H}$ and $\widetilde{E}$, which will be necessarily different of $\widetilde{E}$, the embedding $\widetilde{E} \hookleftarrow \widetilde{E}_{1}$ being compact. And so on.

Remark 4.2 In a first attempt to prove Theorem 1.1 the author tried considering interpolation spaces. More precisely let, for $x \in E$,

$$
K(t, x)=\inf _{a+b=x, a \in E, b \in \mathscr{H}}\left(\|a\|+t|b|_{\mathscr{H}}\right)
$$

and let, for $0<\theta<1$, 


$$
\|x\|_{\theta}=\sup _{t>0} t^{-\theta} K(t, x) .
$$

Let us define the vector space $G_{\theta}$ as the set of vectors $x \in E$ such that $\|x\|_{\theta}<+\infty$, endowed with the norm \|\|$_{\theta}$. See $[1,11]$ or [12] for more details on this topic.

It is well known that $G_{\theta}$ is a Banach space and also that the embeddings $E \hookleftarrow G_{\theta} \hookleftarrow \mathscr{H}$ are compact ([10], §V.2).

The question remains whether $\mu\left(G_{\theta}\right)=1$.

In the case of the Wiener space, $E=\mathscr{C}([0, T], \mathbb{R}), \mathscr{H}=H_{0}^{1}$ and $\mu=$ the Wiener measure, it can be proved that $G_{\theta}$ contains the space of small $\alpha$-Hölder paths for $\alpha>\theta$, which is a separable Banach space having Wiener measure 1 . This gives $\mu\left(G_{\theta}\right)=1$ for $\theta<\frac{1}{2}$, which however leaves open the question in the case $\theta \geq \frac{1}{2}$.

The author does not know whether such a Banach space $G_{\theta}$ is also separable, but it can be proved that the closure of $H_{0}^{1}$ in $G_{\theta}, \widetilde{G}_{\theta}$ say, also contains the small $\alpha$-Hölder paths for $\alpha>\theta$. Hence $\widetilde{G}_{\theta}$, which is separable, is an intermediate space in the sense of Theorem 1.1 in this case. Note that the requirement $\theta<\frac{1}{2}$ means that $G_{\theta}$ should be "closer" to $E$ than to $\mathscr{H}$.

Concerning interpolation spaces, hence, many questions, possibly of interest, remain open. Is it true, in general, that the interpolated space $G_{\theta}$ is an intermediate space? For every $0<\theta<1$ or just for some values of the interpolating parameter $\theta$ ?

Remark 4.3 The construction of the intermediate space $\widetilde{E}$ of Sect. 3 is of course not unique, as other possibilities are available for the candidate norm (3.5). For instance, let us define the sequence $\left(n_{k}\right)_{k}$ so that

$$
\mathrm{E}\left(\left\|\sum_{j=n_{k}+1}^{\infty} g_{j} e_{j}\right\|^{2}\right) \leq 2^{-2 k(\alpha+\eta)} .
$$

for some $\eta>0$ and then, for $x=\sum_{n=1}^{\infty} \alpha_{n} e_{n} \in \mathscr{H}$,

$$
\|x\|^{\prime}=\sup _{k>0} 2^{k \alpha}\left\|\sum_{j=n_{k}+1}^{n_{k+1}} \alpha_{j} e_{j}\right\| .
$$

In this remark we prove that also $\|x\|^{\prime}<+\infty$ for $x \in \mathscr{H}$ and that the completion of $\mathscr{H}$ with respect to \|\|$^{\prime}$ is also an intermediate space.

This has some interest as it shows that there are (many) other possible ways of constructing intermediate spaces. Also we shall see, in the next remark, that for a suitable choice of the orthonormal system $\left(e_{n}\right)_{n}$, in the case $E=\mathscr{C}([0,1])$ and Wiener measure the resulting intermediate spaces are the Hölder spaces.

The proof of $\|x\|^{\prime}<+\infty$ for $x \in \mathscr{H}$ is actually even simpler than the one of Lemma 3.2: let $W_{k}$ as in (3.6) and for $n>0$

$$
Z_{n}=\sup _{k \leq n} 2^{k \alpha}\left\|W_{k}\right\|
$$

Let us show that $Z_{n} \rightarrow Z$ where $Z$ is a r.v. such that $\mathrm{P}(Z<\varepsilon)>0$ for every $\varepsilon>0$. The a.s. convergence of $\left(Z_{n}\right)_{n}$ is immediate being an increasing sequence. Denoting by $Z$ its limit we have 


$$
\begin{aligned}
\mathrm{P}(Z \leq \varepsilon) & =\lim _{n \rightarrow \infty} \mathrm{P}\left(2^{k \alpha}\left\|W_{k}\right\|<\varepsilon, k=1, \ldots, n\right) \\
& =\lim _{n \rightarrow \infty} \prod_{k=1}^{n} \mathrm{P}\left(2^{k \alpha}\left\|W_{k}\right\| \leq \varepsilon\right)=\prod_{k=1}^{\infty}\left(1-\mathrm{P}\left(2^{k \alpha}\left\|W_{k}\right\|>\varepsilon\right)\right) .
\end{aligned}
$$

The infinite product above converges to a strictly positive number if and only if the series $\sum_{k=1}^{\infty} \mathrm{P}\left(2^{k \alpha}\left\|W_{k}\right\|>\varepsilon\right)$ is convergent. But, by Markov's inequality and using the bound $(4.11)$,

$$
\mathrm{P}\left(2^{k \alpha}\left\|W_{k}\right\|>\varepsilon\right) \leq \frac{1}{\varepsilon^{2}} 2^{2 k \alpha} \mathrm{E}\left(\left\|W_{k}\right\|^{2}\right) \leq \frac{1}{\varepsilon^{2}} 2^{-2 k \eta}
$$

which is the general term of a convergent series. Moreover the limit $Z$ is finite a.s., as the r.v.'s $W_{k}$ are independent and the event $\{Z=+\infty\}$ is a tail event having probability $<1$.

The remainder of the proof of Lemma 3.2 is quite similar to the one developed in Sect. 3.

The new norm \|\|$^{\prime}$ also produces a family of intermediate spaces, as stated in the next result

Theorem 4.2 Let $\widetilde{E}^{\prime}=$ the completion of $\mathscr{H}$ with the norm \|\|$^{\prime}$. Then $\widetilde{E}^{\prime}$ is an intermediate space.

Proof Let us prove first that $\mu\left(\widetilde{E}^{\prime}\right)=1$. Let, as in the proof of Theorem 1.1, $Y_{k}=X_{n_{k}}=\sum_{j=1}^{n_{k}} g_{j} e_{j}$ and let us prove that $\left(Y_{k}\right)_{k}$, as a sequence of $\widetilde{E}^{\prime}$-valued r.v.'s, converges in probability. If $k_{0} \leq \ell \leq r$ we have now

$$
\mathrm{P}\left(\left\|Y_{r}-Y_{\ell}\right\|_{i}>\varepsilon\right)=\mathrm{P}\left(\sup _{\ell \leq k \leq r} 2^{\alpha k}\left\|W_{k}\right\|>\varepsilon\right) \leq \mathrm{P}\left(\sup _{k \geq k_{0}} 2^{\alpha k}\left\|W_{k}\right\|>\varepsilon\right) .
$$

Markov's inequality gives $\mathrm{P}\left(2^{\alpha k}\left\|W_{k}\right\|>\varepsilon\right) \leq \frac{1}{\varepsilon^{2}} 2^{-k \eta}$, hence by the Borel-Cantelli Lemma $2^{\alpha k}\left\|W_{k}\right\|>\varepsilon$ for finitely many $k$ only and

$$
\lim _{k_{0} \rightarrow \infty} \mathrm{P}\left(\sup _{k \geq k_{0}} 2^{\alpha k}\left\|W_{k}\right\|>\varepsilon\right)=0
$$

so that $\left(Y_{k}\right)_{k}$ is a Cauchy sequence in probability in $\widetilde{E}^{\prime}$ which implies, with the same argument as in the proof of Theorem 1.1, that $\mu\left(\widetilde{E}^{\prime}\right)=1$.

We are left with the proof that the embedding $\widetilde{E}^{\prime} \hookleftarrow \widetilde{E}$ is compact, which is quite similar to the argument of the proof of Theorem 1.1.

Let again $\left(x_{p}\right)_{p}$ be a bounded sequence in $\widetilde{E}$ and $\left(z_{p}\right)_{p} \subset \mathscr{H}$ another sequence such that $\left\|x_{p}-z_{p}\right\|^{\prime}<2^{-p}$, which is possible as $\mathscr{H}$ is dense in $E$. Let $M$ be such that $\left\|z_{p}\right\|^{\prime} \leq M$ for every $p$. As the projectors $Q_{k}$ are finite dimensional, for every $k$ there exists a subsequence $\left(p_{r}^{(k)}\right)_{r}$ such that $\left\|Q_{k} z_{p_{r}^{(k)}}-y^{(k)}\right\|^{\prime} \rightarrow 0$ for some vector $y^{(k)} \in \mathscr{H}_{k}$, i.e. of the form $y^{(k)}=\sum_{m=n_{k}+1}^{n_{k+1}} \alpha_{m} e_{m}$. By the diagonal argument there exists a subsequence $\left(p_{r}^{\prime}\right)_{r}$ such that $\left\|Q_{k} z_{p_{r}^{\prime}}-y^{(k)}\right\|_{i} \rightarrow 0$ as $r \rightarrow \infty$ for every $k$. Let now $\varepsilon>0$ be fixed. We have for every positive integer $k_{0}$ 


$$
\begin{aligned}
\left\|z_{p_{r}^{\prime}}-z_{p_{\ell}^{\prime}}\right\| & =\left\|\sum_{k=1}^{\infty} Q_{k}\left(z_{p_{r}^{\prime}}-z_{p_{\ell}^{\prime}}\right)\right\| \\
& \leq\left\|\sum_{k=1}^{k_{0}} Q_{k}\left(z_{p_{r}^{\prime}}-z_{p_{\ell}^{\prime}}\right)\right\|+\left\|\sum_{k=k_{0}+1}^{\infty} Q_{k}\left(z_{p_{r}^{\prime}}-z_{p_{\ell}^{\prime}}\right)\right\| .
\end{aligned}
$$

As $\left.\left.\| Q_{k}\left(z_{p_{r}^{\prime}}-z_{p_{\ell}^{\prime}}\right)\right)\left\|\leq 2 \cdot 2^{-k \alpha}\right\| Q_{k}\left(z_{p_{r}^{\prime}}-z_{p_{\ell}^{\prime}}\right)\right) \|^{\prime} \leq 2 \cdot 2^{-k \alpha} M$, we have

$$
\left\|\sum_{k=k_{0}+1}^{\infty} Q_{k}\left(z_{p_{r}^{\prime}}-z_{p_{\ell}^{\prime}}\right)\right\| \leq \sum_{k=k_{0}+1}^{\infty}\left\|Q_{k}\left(z_{p_{r}^{\prime}}-z_{p_{\ell}^{\prime}}\right)\right\| \leq 2 M \sum_{k=k_{0}+1}^{\infty} 2^{-k \alpha} \leq 2^{-k_{0} \alpha} \frac{2 M}{1-2^{-\alpha}}
$$

which, for some $k_{0}$ large enough is $\leq \frac{\varepsilon}{2}$. We can choose now $p_{0}$ so that, for $r, \ell \geq p_{0}$

$$
\left\|\sum_{k=1}^{k_{0}} Q_{k}\left(z_{p_{r}^{\prime}}-z_{p_{\ell}^{\prime}}\right)\right\| \leq \frac{\varepsilon}{2} \text {. }
$$

Therefore $\left(z_{p_{r}^{\prime}}\right)_{r}$ is a Cauchy sequence in $E$, hence also $\left(x_{p_{r}^{\prime}}\right)_{r}$ which proves the compactness of the embedding $\widetilde{E} \hookrightarrow E$.

Note that the norms \|\|$_{i}$ and \|\|$^{\prime}$ differ not only because of their definitions ((3.5) as opposed to (4.12)) but also on the different requirements on the sequence $\left(n_{k}\right)_{k}((3.4)$ and (4.11)).

One of the referees raised the natural question whether the intermediate spaces $\widetilde{E}$ constructed in Theorem 1.1, in the case of the Wiener space might produce the Hölder spaces, or, more generally, if they can be described in terms of regularity of the paths, with the idea that the larger the parameter $\alpha$, the greater the regularity of the paths of $\widetilde{E}$.

The question in general seems to the author to require an analysis going beyond the scope of the present paper, in particular taking into account that regularity also depends on the choice of the orthonormal system $\left(e_{n}\right)_{n}$ and possibly on the regularity of its elements.

In the next remark we show however that, for a certain choice of the orthonormal system, the intermediate spaces of Theorem 4.2 are actually the Hölder spaces $\mathscr{C}_{\alpha}$.

Remark 4.4 Let $E=\mathscr{C}([0,1], \mathbb{R})$ and \|\| the sup norm.

Let us recall the characterization, due to Ciesielski [2], of the small Holder spaces $\mathscr{C}_{\alpha}^{0}$.

Let $\left\{\chi_{n}\right\}_{n}$ be the Haar system, namely the set of functions on the interval $[0,1]$ defined as $\chi_{1}(t) \equiv 1$ and

$$
\chi_{2^{k}+j}(t)= \begin{cases}\sqrt{2^{k}} & \text { if } t \in\left[\frac{2 j-2}{2^{k+1}}, \frac{2 j-1}{2^{k+1}}[\right. \\ -\sqrt{2^{k}} & \text { if } t \in\left[\frac{2 j-1}{2^{k+1}}, \frac{2 j}{2^{k+1}}[\right. \\ 0 & \text { otherwise }\end{cases}
$$

for $k=1,2, \ldots, j=1,2, \ldots, 2^{k}$. It is well known that $\left\{\chi_{n}\right\}_{n}$ is a complete orthonormal system of $L^{2}([0,1], \mathbb{R})$.

Moreover let $\phi_{n}(t)=\int_{0}^{t} \chi_{n}(s) \mathrm{d} s$ be the primitive of $\chi_{n}$ (the Schauder basis) (Fig. 1). For a continuous path $x \in \mathscr{C}([0,1], \mathbb{R})$ let us consider the coefficients $\xi_{n}=\int_{0}^{1} \chi_{n}(s) \mathrm{d} x(s)$ which are well defined, as $\chi_{n}$ is piecewise constant. Ciesielski [2] proved that the separable 


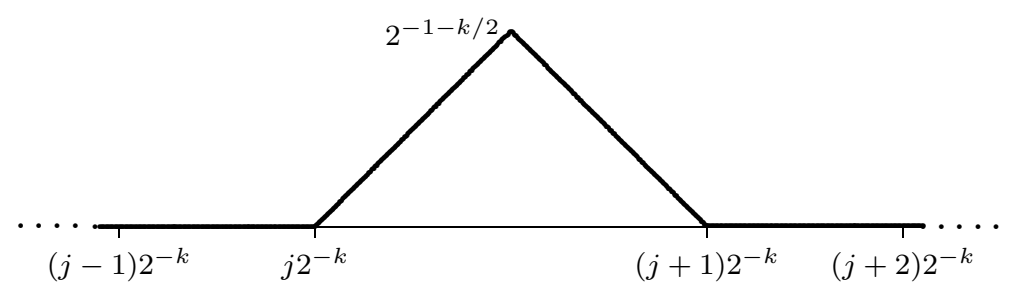

Fig. 1 The graph of $\phi_{2^{k}+j}$

Banach spaces $\mathscr{C}_{\alpha}$ and $c_{0}$ (the sequences vanishing at $\infty$ endowed with the sup norm) are isomorphic $(0<\alpha<1)$.

More precisely if

$$
w_{2^{k}+j}(\alpha)=2^{k\left(\alpha-\frac{1}{2}\right)+(1-\alpha)}
$$

then $x \in \mathscr{C}_{\alpha}, 0<\alpha<1$, if and only if $\xi=\left\{\xi_{n} w_{n}(\alpha)\right\}_{n} \in c_{0}$. Let us denote by $c_{\alpha}$ the space of the sequences $\xi=\left(\xi_{n}\right)_{n}$ such that $\left(\xi_{n} w_{n}(\alpha)\right)_{n} \in c_{0}$. Ciesielski's theorem states that the mapping

$$
\left(\xi_{n}\right)_{n} \leftrightarrow \sum_{m=1}^{\infty} \xi_{m} \phi_{m}
$$

is an isomorphism between $c_{\alpha}$ and $\mathscr{C}_{\alpha}^{0}$.

Remark that under Ciesielski's isomorphism $\mathscr{H}$ is mapped into $\ell_{2}$. Actually $\left(\phi_{n}\right)_{n}$ is itself an orthonormal basis of $\mathscr{H}=H_{0}^{1}$.

The spaces $\mathscr{C}_{\alpha}^{0}$ for $0<\alpha<\frac{1}{2}$ are actually the intermediate spaces obtained in Remark 4.3, if we choose, as an orthonormal system for $\mathscr{H}, e_{n}=\phi_{n}$. Actually, if $n_{k}=2^{k}$ we have, noting that, the supports of the $\phi_{j}$ are disjoint and $\left\|\phi_{j}\right\|=2^{-1-k / 2}$ for $2^{k}+1 \leq j \leq 2^{k+1}$, for large $k$

$$
\mathrm{E}\left(\left\|\sum_{j=2^{k}+1}^{2^{k+1}} g_{j} \phi_{j}\right\|^{2}\right)=2^{-2-k} \mathrm{E}\left(\sup _{2^{k}+1 \leq j \leq 2^{k+1}} g_{j}^{2}\right) \leq 2^{-k \lambda}
$$

for every $\lambda<1$. This is an elementary, but a bit involved, computation that we are not going to explicit here. Hence, as in Remark 4.3, the norm

$$
\|x\|^{\prime}=\sup _{k>0} 2^{k \alpha}\left\|\sum_{j=n_{k}+1}^{n_{k+1}} \alpha_{j} e_{j}\right\|
$$

is finite on $\mathscr{H}$ as soon as $2(\alpha+\eta)<1$, i.e. $\alpha<\frac{1}{2}$. Note, again using the fact that the supports of the $\phi_{j}$ are disjoint and $\left\|\phi_{j}\right\|=2^{-1-k / 2}$ for $2^{k}+1 \leq j \leq 2^{k+1}$, 


$$
\begin{aligned}
& \sup _{k>0} 2^{k \alpha}\left\|\sum_{j=2^{k}+1} \xi_{j} \phi_{j}\right\| \\
& \quad=\sup _{k>0} \sup _{2^{k}+1 \leq j \leq 2^{k+1}} 2^{k \alpha} 2^{-1-k / 2}\left|\xi_{j}\right|=\text { const }|\xi|_{\alpha}
\end{aligned}
$$

so that, thanks to Ciesielski's isomorphism, the intermediate norm \|\|$^{\prime}$ is finite if and only if $x \in \mathscr{C}_{\alpha}^{0}$.

Acknowledgements The author wishes to thank G. Pisier for valuable suggestions and advice. The author wishes to thank one of the referees whose remarks have given rise to significant improvement of the paper. The author acknowledges the MIUR Excellence Department Project awarded to the Dipartimento di Matematica, Università di Roma "Tor Vergata", CUP E83C18000100006.

Funding Open access funding provided by Università degli Studi di Roma Tor Vergata within the CRUICARE Agreement.

Open Access This article is licensed under a Creative Commons Attribution 4.0 International License, which permits use, sharing, adaptation, distribution and reproduction in any medium or format, as long as you give appropriate credit to the original author(s) and the source, provide a link to the Creative Commons licence, and indicate if changes were made. The images or other third party material in this article are included in the article's Creative Commons licence, unless indicated otherwise in a credit line to the material. If material is not included in the article's Creative Commons licence and your intended use is not permitted by statutory regulation or exceeds the permitted use, you will need to obtain permission directly from the copyright holder. To view a copy of this licence, visit http://creativecommons.org/licenses/by/4.0/.

\section{References}

1. Bergh, J., Löfström J.:Interpolation Spaces. An Introduction, Springer, Berlin-New York, Grundlehren der Mathematischen Wissenschaften, No. 223 (1976)

2. Ciesielski, Z.: On the isomorphisms of the spaces $H_{\alpha}$ and $m$. Bull. Acad. Polon. Sci. Sér. Sci. Math. Astronom. Phys. 8, 217-222 (1960)

3. de Acosta, A.: Small deviations in the functional central limit theorem with applications to functional laws of the iterated logarithm. Ann. Probab. 11(1), 78-101 (1983)

4. Donsker, M.D., Varadhan, S.R.S.: Asymptotic evaluation of certain Markov process expectations for large time. III. Commun. Pure Appl. Math. 29(4), 389-461 (1976)

5. Fernique, X.: Intégrabilité des vecteurs gaussiens. C. R. Acad. Sci. Paris Sér. A-B 270, A1698-A1699 (1970)

6. Fernique, X.: Regularité des trajectoires des fonctions aléatoires gaussiennes, École d'Été de Probabilités de Saint-Flour, IV-1974, Springer, Berlin, pp. 1-96. Lecture Notes in Math., Vol. 480 (1975)

7. Gross, L.: Measurable functions on Hilbert space. Trans. Am. Math. Soc. 105, 372-390 (1962)

8. Gross, L.: Abstract Wiener spaces. In: Proceedings of Fifth Berkeley Symposium on Mathematical Statistics and Probability (Berkeley, Calif., 1965/66), Vol. II: Contributions to Probability Theory, Part 1, Univ. California Press, Berkeley, Calif., pp. 31-42 (1967)

9. Ledoux, M., Talagrand, M.: Probability in Banach spaces, Ergebnisse der Mathematik und ihrer Grenzgebiete (3) [Results in Mathematics and Related Areas (3)], vol. 23. Springer, Berlin (1991)

10. Lions, J.-L., Peetre, J.: Sur une classe d'espaces d'interpolation. Inst. Hautes Études Sci. Publ. Math., no. 19, 5-68 (1964)

11. Lunardi, A.: Interpolation theory, Appunti. Scuola Normale Superiore di Pisa (Nuova Serie) [Lecture Notes. Scuola Normale Superiore di Pisa (New Series)], vol. 16, Edizioni della Normale, Pisa (2018)

12. Pisier, G.: Martingales in Banach Spaces. Cambridge Studies in Advanced Mathematics, vol. 155. Cambridge University Press, Cambridge (2016)

Publisher's Note Springer Nature remains neutral with regard to jurisdictional claims in published maps and institutional affiliations. 